psychopraxis. neuropraxis $2018 \cdot 21: 227-231$ https://doi.org/10.1007/s00739-018-0508-9 Online publiziert: 10. September 2018 (c) Der/die Autor(en) 2018

CrossMark

\author{
Henriette Löffler-Stastka ${ }^{1,2} \cdot$ Omar Gelo ${ }^{3} \cdot$ Ingrid Pleschberger ${ }^{1}$. \\ Thomas Schröder ${ }^{4}$ David E. Orlinsky ${ }^{5}$. Michael Helge Rønnestad ${ }^{6}$. \\ Ulrike Willutzki ${ }^{7}$ \\ ${ }^{1}$ Klinik für Psychoanalyse und Psychotherapie, Medizinische Universität Wien, Wien, Österreich \\ ${ }^{2}$ Universitätslehrgang für Psychotherapieforschung, Postgraduate Unit, Teaching Center, Medizinische \\ Universität Wien, Wien, Österreich \\ ${ }^{3}$ University of Lecce, Lecce, Italien \\ ${ }^{4}$ University of Nottingham, Nottingham, Großbritannien \\ ${ }^{5}$ University of Chicago, Chicago, USA \\ ${ }^{6}$ University of Oslo, Oslo, Norwegen \\ ${ }^{7}$ Private Universität Witten/Herdecke, Witten, Deutschland
}

\title{
Psychotherapieausbildung in Österreich
}

\section{Teil 1: Basisdaten und soziodemographische Hintergrunddaten aus der SPRISTAD-Studie}

\section{Zusatzmaterial online \\ In der Online-Version dieses Artikels (https:// doi.org/10.1007/s00739-018-0508-9) finden Sie weiterführende Literatur zum Thema.}

Die Psychotherapieausbildung in Österreich ist vielfältig und diversifiziert. Derzeit gibt es in Österreich 23 anerkannte Psychotherapiemethoden und mehr als 40 Vereine/Ausbildungsinstitute, die an der Ausbildung von Psychotherapeuten beteiligt sind. Die Methoden können nach ihrer Tradition, Schule und Methodik in vier verschiedene Cluster eingeteilt werden, die auf unterschiedlichem theoretischen, philosophisch-erkenntnistheoretischen und anthropologischen Hintergrund basieren: analytisch, humanistisch, systemisch und behavioral.

\section{Psychotherapieausbildung in Österreich}

In Österreich sind die Psychotherapie, die Ausbildung und der Beruf selbst seit 1991 durch ein spezielles Gesetz, das Psychotherapiegesetz, definiert. Das Training, das in diesem Gesetz für zukünftige Psychotherapeuten vorgesehen ist, besteht aus 2 Teilen: einem allge- meinen (Propädeutikum) und einem spezifischen (Fachspezifikum) Teil, beide enthalten theoretische und praktische Elemente.

Die Voraussetzungen, um zum ersten Teil der Ausbildung, dem Propädeutikum, zugelassen zu werden, sind im Vergleich zu anderen deutschsprachigen Ländern offener. Dazu gehören die volle Rechtsfähigkeit der Teilnehmenden, die österreichische Schulabschlussprüfung oder ein anerkanntes Abiturzeugnis aus einem anderen Land oder eine Ausbildung in der Krankenpflege. Erfüllen Teilnehmende diese Voraussetzungen nicht, kann alternativ ein Gutachten des Psychotherapeutenbeirats über deren Tauglichkeit die Eignung nachweisen und zur Zulassung führen.

Um die Zulassung zum zweiten Teil der Ausbildung, dem Fachspezifikum, zu erhalten, muss man voll geschäftsfähig, mindestens 24 Jahre alt sein und das Propädeutikum absolviert haben. Vorliegen muss zudem die schriftliche Zustimmung einer Ausbildungseinrichtung, dass eine Ausbildungsmöglichkeit einschließlich eines Praktikums besteht. Darüber hinaus erfordert eine Zulassung einen Abschluss in den Bereichen Medizin, Pädagogik, Philosophie, Psychologie, Kommunikationswissenschaften, Theo- logie oder Ausbildung zum Lehrer oder Sozialarbeiter. Für den Fall, dass keine dieser "Quellberufe“ ausgeübt wird, besteht die Möglichkeit, einen Sachverständigenbericht über die Qualifikation einzuholen und damit die Zulassung zu erlangen.

Im Vergleich zu anderen Ländern, wie der Schweiz oder Deutschland, wo Psychotherapie vor allem Ärzten und Psychologen vorbehalten ist, hat Österreich den Beruf der Psychotherapeuten einer großen Gruppe von Menschen zugänglich gemacht, mit dem Ziel, das Angebot für die Zielgruppe der Patienten zu erhöhen und die Barrieren für den $\mathrm{Zu}$ gang zu Psychotherapie zu minimieren. Dieser Ansatz erfordert die Durchführung umfassender Ausbildungsprogramme, um sicherzustellen, dass alle Kandidaten, die aus verschiedenen Disziplinen und Berufen kommen, die notwendigen Voraussetzungen für eine effektive psychotherapeutische Behandlung erfüllen.

Das österreichische Psychotherapiegesetz definiert auch das Ausmaß und die Dimensionen der Ausbildung innerhalb des Propädeutikums und des Fachspezifikums. Das Propädeutikum besteht aus einem praktischen und einem theoretischen Teil, den alle Teilnehmenden absolvieren müssen. Der theoretische 
Teil besteht aus mindestens 765 Unterrichtsstunden und muss Themen aus verschiedenen Bereichen abdecken, wie Psychologie, Psychotherapie, Medizin, Psychiatrie, Wissenschafts- und Forschungsmethoden, Ethik sowie soziale und rechtliche Rahmenbedingungen für die Arbeit als Psychotherapeut. Der Praxisteil im Propädeutikum umfasst mindestens 550h Praktikum mit Selbstoder Gruppenselbsterfahrung und eine Supervision, die das Praktikum begleitet. Nach Abschluss des Propädeutikums können Studierende, welche die Voraussetzungen erfüllen, das Fachspezifikum beginnen. Das bedeutet auch, dass sie entscheiden, auf welche therapeutische Methode oder Schule sie sich konzentrieren wollen. Das Fachspezifikum besteht aus einem theoretischen Teil mit $300 \mathrm{Un}$ terrichtsstunden und einem praktischen Teil mit $1600 \mathrm{~h}$ praktischer Erfahrung. Der theoretische Teil ist hier spezialisierter und konzentriert sich auf spezifische Theorien der Entwicklung, Psychopathologie, therapeutische Methoden und Behandlungstechniken sowie das Studium der psychotherapeutischen Literatur. Den Hauptteil des Fachspezifikums bilden jedoch die praktische Erfahrung und die Lehrtherapie. Dadurch soll sichergestellt werden, dass der Auszubildende durch Praktika in psychosozialen, psychiatrischen und psychotherapeutischen Einrichtungen sowie durch zusätzliche Supervision und Lehrtherapie ein individuelles therapeutisches Selbstverständnis erlangt.

Haben die Kandidaten alle diese Anforderungen an Grund- und Fachausbildung (die in der Regel mindestens 5 Jahre dauert) erfüllt, die approbierte Abschlussarbeit verteidigt und das 28 . Lebensjahr vollendet, erhalten sie die österreichische Psychotherapielizenz.

\section{Psychotherapieforschung}

Wie kann eine Person jemanden in emotionaler Not heilen, helfen, einen Sinn im Leben zu finden, lernen, Emotionen $\mathrm{zu}$ verarbeiten oder auszudrücken, und welche Art von Intervention ist für welche Persönlichkeit, Pathologie oder Leidenszustand indiziert? Dies sind Fragen,

psychopraxis. neuropraxis 2018-21:227-231 https://doi.org/10.1007/s00739-018-0508-9 c) Der/die Autor(en) 2018

H. Löffler-Stastka • O. Gelo • I. Pleschberger · T. Schröder · D. E. Orlinsky · M. H. Rønnestad · U. Willutzki

\section{Psychotherapieausbildung in Österreich. Teil 1: Basisdaten und soziodemographische Hintergrunddaten aus der SPRISTAD-Studie}

\section{Zusammenfassung}

Die vorliegende Arbeit stellt einen ersten Einblick in die Ausbildungssituation für Psychotherapeuten in Österreich dar. Auf Grundlage einer Befragung von Kandidaten zu Beginn ihrer Ausbildung werden die Voraussetzungen von Psychotherapieausbildungskandidaten in Österreich betrachtet. Psychotherapeuten wurden am Beginn ihrer Ausbildung in Hinblick auf ihre soziodemografischen Hintergrunddaten und ihren bisherigen Bildungsweg befragt. Diese Hintergrunddaten wurden im Rahmen der internationalen Studie zur Ausbildung und beruflichen Entwicklung von Psychotherapeuten (Society of Psychotherapy Research Interest Section on Therapist Training and Development [SPRISTAD]) mit der Trainee
Background Information Form (TBIF) erfasst. Da es sich um eine explorative Studie handelt, können die Ergebnisse der Hintergrunddaten der bisher 197 Teilnehmenden als Ausgangpunkt für weiterführende Forschung im Bereich Psychotherapieausbildung und Kompetenzentwicklung gesehen werden. Die Ergebnisse werden im Detail demnächst berichtet, sodass weitergehend sowohl auf nationale Bedingungen als auch Möglichkeiten für zukünftige interdisziplinäre Forschung eingegangen werden kann.

\section{Schlüsselwörter}

Interdisziplinäre Forschung · Umfragen und Fragebögen · Ausbildungsunterstützung · Coping · Professionelle Kompetenz

\section{Psychotherapy Training in Austria. Part 1: Baseline and Sociodemographic Background Data from the SPRISTAD Trial}

\section{Abstract}

The present work provides preliminary insight into the training situation for psychotherapists in Austria. On the basis of a survey of candidates at the beginning of their training, the requirements of psychotherapeutic training candidates in Austria are considered. Psychotherapists in training were interviewed at the beginning of their training concerning their sociodemographic background data and prior education. This background data was collected using the Trainee Background Information Form (TBIF), which was designed by the Society for Psychotherapy Research Interest Section on Therapist Training and Development (SPRISTAD). As this is an exploratory study, the results of the background data can be seen as a starting point for further research in psychotherapy training and competence development. Results will be published soon, so that both countrywide conditions and opportunities for future interdisciplinary research can be considered.

\section{Keywords}

Interdisciplinary research · Surveys and questionnaires - Training support · Coping . Professional competence welche die Psychotherapieforschung zu beantworten versucht.

Da es viele Ansätze in der Psychotherapie gibt, wurden verschiedene Aspekte diskutiert: Wirksamkeit und Ergebnis, theoretische Orientierungen, therapeutische Beziehung, Therapeutenfaktoren, Behandlungsformate, Patientencharakteristika, subjektive Parameter und eine Vielzahl allgemeiner Faktoren, die letztendlich zur Formulierung der „Psychotherapy Effectiveness Resolution“ der American Association of Psychology im
Jahr 2012 führte. Ferner haben Forscher begonnen, sich auf die allgemeinen und spezifischen Faktoren zu konzentrieren, die das positive Ergebnis der psychotherapeutischen Behandlung ausmachen, mit anderen Worten: wie und warum Psychotherapie funktioniert. Neue Forschungsimpulse wurden initiiert und neue Perspektiven empirisch geprüft, indem die Therapeutenvariable und die Entwicklung der Therapeuten in den Fokus gerückt wurden. 


\section{Psychotherapieausbildungs- forschung}

Die Psychotherapieforschung widmet sich seit längerem der Identifizierung empirisch gestützter Psychotherapien. Es ist daher naheliegend, dass Wissenschaftler in diesem Bereich zu fragen begannen, welche empirisch unterstützten Faktoren die Entwicklung von Psychotherapeuten fördern. Auch ist mit dem wachsenden Interesse an der Bedeutung der psychotherapeutischen Beziehung das Interesse an Themen der psychotherapeutischen Ausbildungsforschung gestiegen.

\section{》) Was und wie muss jemand lernen, um ein kompetenter Psychotherapeut zu werden?}

Die Frage, was und wie jemand lernen muss, um ein kompetenter Psychotherapeut $\mathrm{zu}$ werden, hat Forscher in den letzten 10 Jahren beschäftigt. Lorentzen, Rønnestad und Orlinsky betonen die Be- deutung von direktem Patientenkontakt, Supervision, Reflexion und Eigentherapie/Selbsterfahrung für die Ausbildung von Therapeuten, und dass diese Charakteristika sich als wichtiger abzeichnen als der Basisberuf. Allerdings müssen ihre Forschungsergebnisse vor dem Hintergrund der Tatsache interpretiert werden, dass alle Therapeuten in der Stichprobe der Studie von Lorentzen et al. eine umfangreiche berufliche Ausbildung entweder als Psychologen oder als Mediziner hatten. Das österreichische Psychotherapieschulensystem bietet ein interessantes Umfeld für Forschungen zur Bedeutung des professionellen Basisberufs für die Kompetenz von Psychotherapeuten, da nicht nur medizinische oder psychologische Fachkräfte die Kriterien erfüllen, um Therapeuten zu werden. Menschen mit unterschiedlichem beruflichen Hintergrund („Quellberufe“) können diesen Beruf nach einer theoretischen und praktischen Ausbildung ausüben.
Wer engagiert sich für die zukünftige Versorgung - welche Kompetenzen bringen Kandidaten mit?

Welche Qualität liegt in der Ausbildung vor? Dies sind Fragen, die im Zusammenhang mit Bedarf-BedürfnisAngebot-Fragen nicht unberücksichtigt bleiben sollten.

Die Bedeutung der Forschung auf dem Gebiet der psychischen Gesundheit und der Behandlung von psychischen Störungen kann nicht überschätzt werden. Verbunden mit der Frage, was ein kompetenter Psychotherapeut braucht und ob die Psychotherapieausbildung die Entwicklung der Teilnehmenden fördert, sollte die Forschung interdisziplinäre Ansätze und Konzepte berücksichtigen.

\section{Ziele}

Als Antwort auf den großen Bedarf an Langzeitstudien zur Psychotherapieausbildung initiierte die Forschungsgruppe für Therapeutenausbildung und -ent-

Hier steht eine Anzeige. 


\begin{tabular}{l|l|l|}
\hline Tab. 1 SPRISTAD, Kerninstrumente & Beschreibung & Erhebungszeitpunkte \\
\hline Instrument & $\begin{array}{l}\text { Fragebogen zu Struktur und Inhalt des Ausbil- } \\
\text { dungsprogramms }\end{array}$ & $\begin{array}{l}\text { Ein Messzeitpunkt (Beginn der Beteiligung } \\
\text { an der Studie) }\end{array}$ \\
\hline $\begin{array}{l}\text { TPDF Training Program Description Form } \\
\text { zur Ausbildungsordnung }\end{array}$ & $\begin{array}{l}\text { Fragebogen zu Hintergrunddaten der Ausbildungs- } \\
\text { kandidaten inklusive soziodemografische Daten, } \\
\text { aktuelle Lebenssituation, eigene Therapieerfah- } \\
\text { rung: Selbstwahrnehmung und Persönlichkeit }\end{array}$ & $\begin{array}{l}\text { Ein Messzeitpunkt (Beginn der Beteiligung } \\
\text { an der Studie) }\end{array}$ \\
$\begin{array}{l}\text { Kandidaten } \\
\text { TCackground Information Form }\end{array}$ & $\begin{array}{l}\text { Fragebogen an Kandidaten zu generellen Erfahrun- } \\
\text { gen in der aktuellen Ausbildungspraxis }\end{array}$ & $\begin{array}{l}\text { Wiederholte Messzeitpunkte alle 6 Monate } \\
\text { (mindestens 3 Zeitpunkte) }\end{array}$ \\
\hline $\begin{array}{l}\text { Kandidaten } \\
\text { SPRISTAD Society of Psychotherapy Research Interest Section on Therapist Training and Development }\end{array}$ & \\
\hline
\end{tabular}

Tab. 2 SPRISTAD, optionale Instrumente

\begin{tabular}{|c|c|c|}
\hline Instrument & Beschreibung & Erhebungszeitpunkte \\
\hline $\begin{array}{l}\text { TCPR/c Trainee Case Progress Report } \\
\text { Kandidat }\end{array}$ & $\begin{array}{l}\text { Adaptierung des TCPR mit Fokus auf die Erfahrung } \\
\text { des Kandidaten mit einem spezifischen Fall }\end{array}$ & $\begin{array}{l}\text { Wiederholte Messzeitpunkte (freiwillige } \\
\text { Festlegung) }\end{array}$ \\
\hline $\begin{array}{l}\text { TSPR Trainee Supervisor's Progress Report } \\
\text { Supervisor }\end{array}$ & $\begin{array}{l}\text { Adaptierung des TCPR mit Fokus auf die Erfahrung } \\
\text { des Supervisors mit einem spezifischen Fall }\end{array}$ & $\begin{array}{l}\text { Wiederholte Messzeitpunkte (freiwillige } \\
\text { Festlegung) }\end{array}$ \\
\hline
\end{tabular}

wicklung (Interest Section on Therapist Training and Development) der Society of Psychotherapy Research eine Multi-Site-Longitudinalstudie (SPRISTADStudie) zur Entwicklung von Psychotherapeuten. Sie zielt darauf ab, den progressiven Wandel im Laufe der Zeit bei Ausbildungskandidaten zu verfolgen, um Faktoren $\mathrm{zu}$ identifizieren, welche die Entwicklung von Auszubildenden fördern oder erschweren, indem quantitative und qualitative Daten aus einer Vielzahl von Ausbildungsprogrammen gesammelt werden.

Eine solche internationale Perspektive ist besonders relevant, da lokale Forschungsprojekte häufig mit Einschränkungen hinsichtlich ihrer Stichprobengröße und ihrer Ressourcen konfrontiert sind, denn die Anzahl der Auszubildenden in den einzelnen Ausbildungsprogrammen ist eher klein.

Inspiriert durch Multi-Site-Studien in der Psychotherapieforschung umrahmte der SPRISTAD-Lenkungsausschuss die Ziele für die Studie als:

- Verfolgung progressiver Veränderungen im Laufe der Zeit bei Psychotherapiekandidaten,

- Identifizierung von Faktoren, die dazu beitragen, die Entwicklung von Auszubildenden zu fördern oder zu erschweren und

- das Erreichen der o.g. Ziele mithilfe von quantitativen und qualitativen
Daten, die von einer großen Anzahl von Psychotherapeuten verschiedener Typen in einem breiten Spektrum von Ausbildungsprogrammen gesammelt wurden.

Als erster Schritt für Studien in diesen Bereichen werden die Charakteristika von Ausbildungskandidaten zu Beginn ihrer psychotherapeutischen Arbeit betrachtet - in dieser Arbeit zum Beginn des Fachspezifikums.

\section{Methodik}

Die SPRISTAD-Studie ist weltweit angelegt. Regionale und lokale Koordinatoren für Forschungsstandorte organisieren und verteilen Informationen in (derzeit) 18 Ländern, die Interesse an der Teilnahme an der Studie bekundet haben.

\section{Instrumente}

Die Instrumente umfassen mehrere Online-Fragebögen, die in obligatorische Kerninstrumente (- Tab. 1) und Instrumente zur freiwilligen Anwendung unterteilt sind (•Tab. 2).

Zwei optionale Fragebögen (• Tab. 2) werden parallel zu den Kerninstrumenten eingesetzt, um Informationen und Sichtweisen von Kandidaten und deren Supervisoren zu erheben.

\section{Die SPRISTAD-Studie in Österreich}

Die aktuell hier vorliegende Studie stellt die erste Erhebung der SPRISTAD-Studie in Österreich dar. Methodisch und die Verwendung der Instrumente betreffend ist die Befragung in Österreich dementsprechend identisch mit der weltweiten SPRISTAD-Studie. Sie ist als eine systematische explorative Untersuchung definiert, die eine realistische Darstellung der Entwicklung von Auszubildenden ermöglichen soll und die Art der Ausbildungserfahrungen erkennen lässt, die signifikant und differenziell mit der beruflichen Entwicklung von Therapeuten in der Ausbildung zusammenhängen und mit erfahrungsbezogenen und soziodemographischen Hintergrundinformationen verknüpft werden können.

Alle Kandidaten der SPRISTAD-Studie in Österreich nahmen freiwillig an der Studie teil und haben schriftlich zugestimmt, dass ihre anonymisierten Daten $\mathrm{zu}$ wissenschaftlichen Zwecken verwendet und veröffentlicht werden können. Sie wurden darüber informiert, dass die Studie dazu beitragen soll, wichtige Faktoren für die Psychotherapieausbildung herauszufinden, und dazu anregt, sich mit der persönlichen Geschichte auseinanderzusetzen. Alle wurden gebeten, einen Fragebogen zu ihren Hintergrunddaten auf der Grundlage des in der SPRI- 
STAD-Studie verwendeten Fragebogens auszufüllen (•Tab. 1). Die hier berichteten ersten Befragungen der SPRISTADStudie in Österreich wurden gesamt von der Ethikkommission der Medizinischen Universität Wien als Leitethikkommission geprüft und erhielten ein positives Votum. Die in - Tab. 1 und 2 dargestellten Instrumente wurden einheitlich für die Deutsch sprechenden Teilnehmenden übersetzt.

\section{Hintergrundinformation}

Die Fragebögen zur Hintergrundinformation der Ausbildungskandidaten wurden als Trainee Background Information Form (TBIF) entwickelt, die Teil des SPRISTAD-Projekts ist. Ein Überblick ist in - Tab. 1 gegeben. Ein 27-Punkte-Online-Fragebogen zur Erfassung von soziodemographischen Daten, wie Alter, Geschlecht, Nationalität, Bildung und sozioökonomischem Status in der Herkunftsfamilie/Kindheit, sowie von Informationen über die Ausbildungseinrichtung, in der die Psychotherapieausbildung absolviert wird, diente dazu, ein umfassendes Profil der Kohorte zu bekommen.

\section{Ergebnisse und Diskussion}

In dieser Studie wurden im Kontext der Internationalen Studie zu Training und beruflicher Entwicklung von Psychotherapeuten (SPRISTAD-Studie) insgesamt bisher 197 Ausbildungskandidaten zu Beginn ihres Fachspezifikums bezüglich ihrer demographischen Daten und beruflichen Vorerfahrungen befragt. Die Ergebnisse werden aktuell ausgearbeitet und demnächst publiziert. Insgesamt stellen die vorläufigen Ergebnisse dieser Arbeit eine erste Basis für weitere Forschungen dar. Aufgrund des Multi-Site-Designs in der SPRISTAD-Studie insgesamt und in Österreich ist eine hohe Teilnehmendenzahl zu erwarten; international und spezifisch für Österreich sind zahlreiche weiterführende Fragestellungen möglich. Auch könnte exploriert werden, ob manche soziodemographischen Faktoren als moderierende Variablen dienen.

Die vorliegende Studie stellt einen ersten Startpunkt zur empirischen Befor- schung der subjektiven Charakteristika der Therapeuten dar. Weitergehende Arbeiten werden auf dieser Basis Einblick geben in die Relevanz von kulturellen Haltungen, Wertsystemen und Überzeugungen, in die Relevanz von Lebensqualität, emotionaler Zufriedenheit, Persönlichkeit und Coping-Strategien der Therapeuten wie der Auszubildenden. Sofern sich viele Ausbildungskandidaten bereiterklären, Prozess- und Ergebnisdaten der von ihnen durchgeführten Therapien auf ihre Merkmale vor und während der Ausbildung zu beziehen, kann deren Relevanz für den Therapieerfolg untersucht werden und damit kann die Versorgungssituation reflektierbar, diskutierbar und planbar gemacht werden.

\section{Fazit für die Praxis}

- Da mittlerweile viele unterschiedliche Orientierungen in Österreich und international an der Studie beteiligt sind, könnte die Studie auch Einblick in die unterschiedlichen therapeutischen Traditionen, in Methodensozialisation und Erwartungen sowie in unterschiedliche therapeutische Formen der Beziehungsgestaltung geben (TCPR[Trainee Current Practice Report]-Fragebogen).

- Beispielsweise können durch Kombination mit anderen Interviewverfahren Untersuchungen durchgeführt werden, wie in anderen Pilotprojekten bereits berichtet wurde.

\section{Korrespondenzadresse}

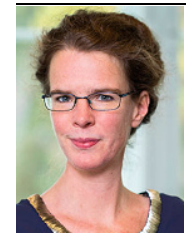

Prof. Dr. Henriette LöfflerStastka

Klinik für Psychoanalyse und Psychotherapie, Medizinische Universität Wien

Währinger Gürtel 18-20,

() Medizinische Universität Wien 1090 Wien, Österreich henriette.loefflerstastka@meduniwien.ac.at

Danksagung. Wir danken allen Forschungsbeauftragten der Vereine und Ausbildungsinstitute und allen Teilnehmenden.

Funding. Open access funding provided by Medical University of Vienna.
Einhaltung ethischer Richtlinien

Interessenkonflikt. H. Löffler-Stastka, O. Gelo, I. Pleschberger, T. Schröder, D.E. Orlinsky, M.H. Rønnestad und U. Willutzki geben an, dass kein Interessenkonflikt besteht.

Dieser Beitrag beinhaltet keine von den Autoren durchgeführten Studien an Patienten oder Tieren. Die Ethikkommission der Medizinischen Universität Wien prüfte und befürwortete die Studie, die schriftlichen Einverständniserklärungen der Teilnehmer sowie de teilnehmenden Ausbildungsinstitute wurden nach informed consent eingeholt und liegen vor.

Open Access. Dieser Artikel wird unter der Creative Commons Namensnennung 4.0 International Lizenz (http://creativecommons.org/licenses/by/4.0/deed. de) veröffentlicht, welche die Nutzung, Vervielfältigung, Bearbeitung, Verbreitung und Wiedergabe in jeglichem Medium und Format erlaubt, sofern Sie den/die ursprünglichen Autor(en) und die Quelle ordnungsgemäß nennen, einen Link zur Creative Commons Lizenz beifügen und angeben, ob Änderungen vorgenommen wurden. 